\title{
Prevalence and predictors of medication non-adherence in some common non-communicable chronic diseases and cancers: a comparative study
}

\author{
Shakeel A. Mir ${ }^{1 *}$, Danish Shakeel ${ }^{2}$ \\ ${ }^{1}$ Department of Clinical Pharmacology, Sher-I-Kashmir Institute of Medical Sciences, Srinagar, Kashmir, India \\ ${ }^{2}$ Department of Computer Science and Engineering, Apex Institute of Engineering, Chandigarh University, Mohali, \\ Punjab, India
}

Received: 29 August 2020

Accepted: 05 October 2020

*Correspondence:

Dr. Shakeel A. Mir,

Email: drshakeelahmadmir@gmail.com

Copyright: $@$ the author(s), publisher and licensee Medip Academy. This is an open-access article distributed under the terms of the Creative Commons Attribution Non-Commercial License, which permits unrestricted non-commercial use, distribution, and reproduction in any medium, provided the original work is properly cited.

\begin{abstract}
Background: Adherence is a key factor in the success of all pharmacological therapies. Medication non-adherence is an extremely common barrier to achieve positive health outcomes. The present study aims to compare medication non-adherence in some common chronic diseases and cancers and also to assess various factors influencing it.

Methods: Pre-validated questionnaires based on general medication adherence scale (GMAS) were equally distributed among 300 patients suffering from chronic illnesses and cancers. 270 patients returned completely filled questionnaires.

Results: The study population consists of $53.33 \%$ men and $46.66 \%$ women.55.55\% patients were literate. $70.37 \%$ of patients were from rural areas. $61.48 \%$ were taking 2-4 drugs. As compared to $14.81 \%$ males $18.51 \%$ of females had poor or low adherence. Only $0.74 \%$ of young patients (<30 years) had poor/low adherence as compared to $16.29 \%$ each in other age groups. Rural patients had poor adherence as compared to patients from urban areas. As compared to $31.85 \%$ illiterate patients, only $16.29 \%$ of literate patients had poor or low adherence. Adherence was better in patients taking more than one drug. Statistically, a significant correlation was found between gender, age, level of education, and area of residence. Duration of treatment, the number of drugs, and the frequency of dosage were not found significantly correlated with adherence. Patients suffering from malignancies had higher medication adherence as compared to chronic illnesses.

Conclusions: Medication non-adherence is common in patients with chronic diseases and cancers that are treated with unsupervised oral antineoplastic drugs. The complex problem of non-adherence calls for interventions at various levels.
\end{abstract}

Keywords: Medication, Adherence, Oral antineoplastics, Treatment, Cancers, Chronic diseases

\section{INTRODUCTION}

The WHO defines adherence to long term therapies as the extent to which a person`s behavior-taking medication, following a diet and/or executing lifestyle changescorrespond with agreed recommendations from a healthcare provider. ${ }^{1}$ Poor adherence to prescribed regimens leads to worsening of the disease, and increased health care costs and even death. ${ }^{2}$ Medication nonadherence annually costs from US\$ 100 to U\$ 290 billion in the USA, $€ 1.25$ billion in Europe and approximately \$A7 billion in Australia. ${ }^{3}$ 
Poor medication adherence in chronic diseases is a worldwide problem. Approximately $50 \%$ of patients do not adhere to one of their chronic medications. ${ }^{1}$

Recently the availability of oral anticancer drugs has increased significantly. But simultaneously concerns about adherence have become an important issue, especially with unsupervised chronic oral therapy. ${ }^{4}$ Although patients with cancers exhibit higher motivation towards medication adherence, adherence ranges from $16 \%$ to $100 \% .^{5}$

When the treatment is carried out without direct supervision of the medical personnel, medication adherence often becomes an issue adversely affecting the effectiveness of the therapy. ${ }^{6}$

There are a small number of studies on comparisons of drug non-adherence across different diseases. Most of the studies have focused on a single disease. Our study aims to compare medication non-adherence in some common chronic non-communicable diseases and malignancies and also to assess various predictors.

There are various methods to assess medication adherence. These include directly observed therapy, biological methods, and serum or urinary drug levels. Other methods include clinician reports, pill counts, rates of prescription refills, electronic medication monitors, patient diaries, and patient self-reports. ${ }^{7}$

\section{METHODS}

Direct methods are expensive and burdensome. ${ }^{8}$ We used the self-report method. Self-report measures have the benefit of being relatively simple, quick, cheap, reliable, valid, non-intrusive and easy to administer. ${ }^{7}$

\section{Study design}

This is a descriptive questionnaire-based study, conducted in a period of one year from March 2019 to March 2020, in SKIMS Hospital Srinagar, Kashmir. A sample size calculation was done to determine the population required for the study.

\section{Sample size calculation}

$$
\mathrm{n}=\mathrm{pq} /(\mathrm{E} / 1.96) 2 \text { where }
$$

$\mathrm{n}=$ minimum sample size, $\mathrm{p}=$ prevalence of non-adherence in previous studies $(25 \%), \quad q=100-p(100-25)=75$, $\mathrm{E}=$ margin of sample error tolerated $(\%)=5 \%$ i.e $95 \%$ confidence interval,

$\mathrm{n}=75 \times 25(5 / 1.96) 2=288$

(288 was the sample size required. We randomly selected 300 patients, 150 from each group).

\section{Study population}

The study population consisted of two types of patients. Group one comprised of the patients suffering from common chronic non-communicable diseases like hypertension, rheumatoid arthritis, chronic obstructive Pulmonary Disease, bronchial asthma, parkinsonism, chronic renal disease, hyperlipidemia, and diabetes mellitus. Group two comprised of patients suffering from malignancies involving the liver, breast, colon, lung, stomach and prostate. We included only those cancer patients taking at least one oral anticancer drug. But adherence measurements included all the drugs the patient was taking concomitant to an anticancer drug. Patients attending follow up clinics were enrolled for the study.

A total of 150 patients from each group were given the questionnaire. All the 150 patients from group two returned the completely filled questionnaire. But from group one, only 130 returned the questionnaire, out of which only 120 questionnaires had been filled up completely.

\section{Study tools}

A pre-tested and validated questionnaire that was prepared in english and urdu. It contained three sections. Section first included the questions regarding the general demographic information such as age, gender, location, and educational qualification. Section two had questions about disease and medication. Section three asked questions about medication adherence. We used the English and Urdu versions of a novel medication adherence tool known as GMAS (general medication adherence scale), after obtaining permission from the authors of the Scale. ${ }^{9}$ The scale categorizes adherence into poor, low, partial, good, and high.

\section{Sampling method}

Simple random sampling

\section{Consents}

The participants were informed about study objectives before data collection and questionnaires were filled only by those who were willing to fill it. Written informed consent was obtained from all the participants. The confidentiality of the participants was maintained.

\section{Inclusion criteria}

Inclusion criteria were suffering from well-established chronic non-communicable disease or cancer, taking drugs for the minimum last six months, cancer patients receiving at least one unsupervised oral anticancer drug, age above 18 years, willing to participate in the study, 
ability to communicate by at least one of the means viz. speaking or writing

\section{Exclusion criteria}

Exclusion criteria were suffering from acute diseases, taking drugs for less than six months, age below 18 years, unwilling to participate in the study, unable to communicate by at least one of the means viz. speaking or writing.

\section{Statistical tools}

Analysis was done by a combination of manual calculators and free online statistics software "Vassar Stats".

\section{RESULTS}

There were 120 patients in chronic diseases group (Group 1), with $46.7 \%(n=56)$ male and $53.3 \%(n=64)$ female. In cancer group, (Group 2) there were 150 patients, with $58.7 \%(n=88)$ male and $41.3 \%(n=62)$ female. In chronic disease group, $35.0 \%(\mathrm{n}=42)$ were literate and $65.0 \%$ $(\mathrm{n}=78)$ were illiterate. In cancer group, $52.0 \% \quad(\mathrm{n}=78)$ were literate and $48.0 \%(n=72)$ were illiterate. In chronic disease group, $75.00 \%(\mathrm{n}=90)$ were from rural areas and $25.00 \%(\mathrm{n}=30)$ were from urban areas. In cancer group, $66.7 \%(n=100)$ were from rural areas and $33.3 \%(n=50)$ were from urban areas. In chronic disease group, $11.6 \%$ $(n=14)$ patients were less than 30 years, $45.0 \%(n=54) 30$ 60 years, and $43.4 \%(n=52)$ patients above 60 years. In cancer group, $5.3 \%(\mathrm{n}=8)$ patients were less than 30 years old, $66.7 \%(n=100) 30-60$ years, and $28.0 \%(n=42)$ were above 60 years (Table 1$)$.

Table 1: Demographics of the participants.

\begin{tabular}{|c|c|c|c|c|c|c|c|}
\hline \multirow{3}{*}{ Group } & \multicolumn{7}{|c|}{ Gender } \\
\hline & \multicolumn{3}{|c|}{ Male } & \multicolumn{3}{|c|}{ Female } & \multirow[t]{2}{*}{ Total } \\
\hline & $\mathbf{N}$ & $\%$ & & $\mathbf{N}$ & $\%$ & & \\
\hline Chronic diseases & 56 & 46.66 & & 64 & & & 120 \\
\hline Cancers & 88 & 58.66 & & 62 & & & 150 \\
\hline \multirow{2}{*}{ Group } & \multicolumn{7}{|c|}{ Literacy } \\
\hline & \multicolumn{3}{|c|}{ Literate } & \multicolumn{3}{|c|}{ Illiterate } & Total \\
\hline Chronic diseases & 42 & 35.00 & & 78 & & & 120 \\
\hline Cancers & 78 & 52.00 & & 72 & & & 150 \\
\hline \multirow{2}{*}{ Group } & \multicolumn{7}{|c|}{ Residence } \\
\hline & \multicolumn{3}{|c|}{ Rural } & \multicolumn{3}{|c|}{ Urban } & Total \\
\hline Chronic diseases & 90 & 75.00 & & 30 & & & 120 \\
\hline Cancers & 100 & 66.66 & & 50 & & & 150 \\
\hline \multirow{3}{*}{ Group } & \multicolumn{7}{|c|}{ Age-wise distribution } \\
\hline & \multicolumn{2}{|c|}{$<30$ yrs } & \multicolumn{2}{|c|}{ 30-60 years } & \multicolumn{2}{|c|}{$>60$ years } & Total \\
\hline & No & $\%$ & No & $\%$ & No & $\%$ & \\
\hline Chronic diseases & 14 & 11.66 & 54 & 45.00 & 52 & 43.33 & 120 \\
\hline Cancers & 8 & 5.33 & 100 & 66.66 & 42 & 28.00 & 150 \\
\hline
\end{tabular}

Table 2: Pattern of disease and drug use.

\begin{tabular}{|c|c|c|c|c|c|c|}
\hline \multirow{3}{*}{ Group } & \multicolumn{6}{|c|}{ Duration of treatment } \\
\hline & \multicolumn{2}{|c|}{$<1$ year } & \multicolumn{2}{|c|}{ 1-4 year } & \multicolumn{2}{|c|}{$>4$ year } \\
\hline & $\mathbf{N}$ & $\%$ & $\mathbf{N}$ & $\%$ & $\mathbf{N}$ & $\%$ \\
\hline Chronic diseases & 20 & 16.7 & 60 & 50.00 & 40 & 33.3 \\
\hline Cancers & 32 & 21.3 & 98 & 65.33 & 20 & 13.6 \\
\hline \multirow{3}{*}{ Group } & \multicolumn{6}{|c|}{ Number of prescribed drugs } \\
\hline & \multicolumn{2}{|c|}{ One drug } & \multicolumn{2}{|c|}{ 2-4 drugs } & \multicolumn{2}{|c|}{$>4$ drugs } \\
\hline & No & $\%$ & No & $\%$ & No & $\%$ \\
\hline Chronic diseases & 48 & 40.0 & 64 & 53.3 & 8 & 6.7 \\
\hline Cancers & 20 & 13.4 & 102 & 68.0 & 28 & 18.6 \\
\hline \multirow{3}{*}{ Group } & \multicolumn{6}{|c|}{ Frequency of drug administration } \\
\hline & \multicolumn{2}{|c|}{ Once a day } & \multicolumn{2}{|c|}{ Twice a day } & \multicolumn{2}{|c|}{ Thrice a day or more } \\
\hline & No & $\%$ & No & $\%$ & No & $\%$ \\
\hline Chronic diseases & 52 & 43.3 & 36 & 30.0 & 32 & 26.7 \\
\hline Cancers & 50 & 33.3 & 52 & 34.87 & 48 & 32.0 \\
\hline
\end{tabular}


In chronic disease group, $16.7 \%(\mathrm{n}=20)$ patients were on treatment for less than one year, $50.0 \%(n=60)$ for $1-4$ years, and $33.3 \%(\mathrm{n}=40)$ for more than four years. In cancer group, $21.3 \%(\mathrm{n}=32)$ were on treatment for less than a year, $65.33 \%(\mathrm{n}=98)$ for $1-4$ years, and $13.6 \%$ $(n=20)$ for more than 4 years. In chronic disease group, $40.0 \%(\mathrm{n}=48)$ patients received one drug, 53.3\% $(\mathrm{n}=64)$ 2-4 drugs, and $6.7 \%(n=8)$ more than four drugs a day. In cancer group, $13.4 \%(\mathrm{n}=20)$ patients received one drug, $68.0(n=102) 2-4$ drugs, and $18.6 \%(n=28)$ more than 4 drugs a day. In chronic disease group, $43.3 \%(n=52)$ were taking drugs once a day, $30.0 \%(n=36)$ twice a day, and $26.7 \%(n=32)$ thrice a day or more. In cancer group, $33.3 \%(n=50)$ were taking drug once a day, $34.87 \%$ $(n=52)$ twice a day and $32.0 \%(n=48)$ thrice a day or more (Table 2).

In chronic disease group, as per the GMAS used, $14.8 \%$ males $(n=8)$ had poor, $53.57 \%(n=30)$ low, 14.28\% $(n=8)$ partial, and $17.85 \%(\mathrm{n}=10)$ good adherence. Among females, $14.06 \%(n=9)$ had poor, $45.31 \%(n=29)$ low, $18.75 \%(\mathrm{n}=12)$ partial, $18.75 \%(\mathrm{n}=12)$ good, and $3.12 \%$ $(\mathrm{n}=2)$ high adherence. In cancer group, $1.13 \%(\mathrm{n}=1)$ male had poor, $1.13 \%(n=1)$ low, $15.90 \% \quad(n=14)$ partial,
$34.09 \%(n=30)$ good and $47.72 \%(n=42)$ high adherence. Among females, 6.45\% $(\mathrm{n}=4)$ had poor, $12.90 \%(\mathrm{n}=8)$ low, $16.12 \%(n=10)$ partial, $48.38 \%(n=30)$ good and $16.12 \%(n=10)$ high adherence. In chronic disease group, those less than 30 years $42.85 \%(n=6)$ had partial, $42.85 \%$ $(n=6)$ good and $14.28 \%(n=2)$ high adherence. In 30-60 years, subgroup, $18.51 \%(\mathrm{n}=10)$ had poor, $40.74 \%(\mathrm{n}=22)$ low, $18.51 \% \quad(n=10)$ partial, $22.22 \% \quad(n=12)$ good adherence. In above 60 sub group, $21.15 \%(n=11)$ had poor, $63.46 \%(\mathrm{n}=33)$ low, $7.69 \%(\mathrm{n}=4)$ partial, and $7.69 \%$ $(n=4)$ good adherence. In cancer group, those below 30 years had, $12.50 \%(\mathrm{n}=1)$ poor, $12.50 \%(\mathrm{n}=1)$ low, $25.00 \%$ $(n=2)$ partial, $25.00 \%(n=2)$ good and $25.00 \%(n=2)$ high adherence. In 30-60 yr sub group, $4.00 \%(\mathrm{n}=4)$ had poor, $8.0 \%(n=8)$ low, $14.00 \%(n=14)$ partial, $40.00 \%(n=40)$ good and $34.00 \%(n=34)$ high adherence. In above 60 year sub group, $14.28 \%(n=6)$ had partial, $47.61 \%(n=20)$ good and $38.09 \%(\mathrm{n}=16)$ high adherence. In chronic disease group, $33.3 \%(n=26)$ illiterate had poor, $64.10 \%$ $(n=50)$ low, $1.28 \%(n=1)$ partial, and $1.28 \%(n=1)$ good adherence. Among literate patients, $23.80 \%(\mathrm{n}=10)$ had poor, $71.42 \%(\mathrm{n}=30)$ low, $2.38 \%(\mathrm{n}=1)$ partial, and $2.38 \%$ $(\mathrm{n}=1)$ good adherence.

Table 3: GMAS Score in different groups.

\begin{tabular}{|c|c|c|c|c|c|c|}
\hline & Gender & Poor & Low & Partial & Good & High \\
\hline \multirow{2}{*}{ Ch diseases } & Male & $8(14.28)$ & $30(53.57)$ & $8(14.28)$ & $10(17.85)$ & $0(0.00)$ \\
\hline & female & $9(14.06)$ & $29(45.31)$ & $12(18.75)$ & $12(18.75)$ & $2(3.12)$ \\
\hline \multirow{3}{*}{ Cancers } & male & $1(1.13)$ & $1(1.13)$ & $14(15.90)$ & $30(34.09)$ & $42(47.72)$ \\
\hline & female & $4(6.45)$ & $8(12.90)$ & $10(16.12)$ & $30(48.38)$ & $10(16.12)$ \\
\hline & Age & Poor & Low & Partial & Good & High \\
\hline \multirow{3}{*}{ Ch diseases } & $<30$ & $0(0.0)$ & $0(0.0)$ & $6(42.85)$ & $6(42.85)$ & $2(14.28)$ \\
\hline & $30-60$ & $10(18.51)$ & $22(40.74)$ & $10(18.51)$ & $12(22.22)$ & $0(0.00)$ \\
\hline & $>60$ & $11(21.15)$ & $33(63.46)$ & $4(7.69)$ & $4(7.69)$ & $0(0.00)$ \\
\hline \multirow{4}{*}{ Cancers } & $<30$ & $1(12.5)$ & $1(12.5)$ & $2(25.00)$ & $2(25.00)$ & $2(25.00)$ \\
\hline & $30-60$ & $4(4.00)$ & $8(8.00)$ & $14(14.00)$ & $40(40.00)$ & $34(34.00)$ \\
\hline & $>60$ & $0(0.00)$ & $0(0.00)$ & $6(14.28)$ & $20(47.61)$ & $16(38.09)$ \\
\hline & Literacy & Poor & Low & Partial & Good & High \\
\hline \multirow{2}{*}{ Ch disease } & Illiterate & $26(33.33)$ & $50(64.10)$ & $1(1.28)$ & $1(1.28)$ & $0(0.0)$ \\
\hline & Literate & $10(23.80)$ & $30(71.42)$ & $1(2.38)$ & $1(2.38)$ & $0(0.0)$ \\
\hline \multirow{3}{*}{ Cancers } & Illiterate & $3(3.65)$ & $7(8.53)$ & $17(20.73)$ & $35(42.68)$ & $20(24.39)$ \\
\hline & literate & $1(1.47)$ & $3(4.41)$ & $10(14.70)$ & $22(32.35)$ & $32(47.05)$ \\
\hline & Location & Poor & Low & Partial & Good & High \\
\hline \multirow{2}{*}{ Ch Diseases } & rural & $25(27.77)$ & $51(56.66)$ & $6(6.66)$ & $8(8.88)$ & $0(0.0)$ \\
\hline & urban & $0(0.00)$ & $0(0.00)$ & $10(33.33)$ & $18(60.00)$ & $2(6.77)$ \\
\hline \multirow{3}{*}{ Cancers } & rural & $2(2.00)$ & $10(10.00)$ & $8(8.00)$ & $50(50.00)$ & $30(30.00)$ \\
\hline & urban & $0(0.00)$ & $0(0.00)$ & $8(16.00)$ & $20(40.00)$ & $22(44.00)$ \\
\hline & Rx Duration & Poor & Low & Partial & Good & High \\
\hline \multirow{3}{*}{ Ch diseases } & $<1 \mathrm{yr}$ & $5(25.00)$ & $15(75.00)$ & $0(0.00)$ & $0(0.00)$ & $0(0.00)$ \\
\hline & $1-4 \mathrm{yr}$ & $16(26.66)$ & $40(66.66)$ & $2(3.33)$ & $2(3.33)$ & $0(0.00)$ \\
\hline & $>4 \mathrm{yr}$ & $0(0.00)$ & $0(0.00)$ & $10(25.00)$ & $28(70.00)$ & $2(5.00)$ \\
\hline \multirow{3}{*}{ Cancers } & $<1 \mathrm{yr}$ & $1(3.12)$ & $1(3.12)$ & $4(12.50)$ & $16(50.00)$ & $10(32.25)$ \\
\hline & $1-4 \mathrm{yr}$ & $4(4.08)$ & $8(8.16)$ & $10(10.20)$ & $40(40.81)$ & $36(36.73)$ \\
\hline & $>4 \mathrm{yr}$ & $0(0.00)$ & $0(0.00)$ & $4(20.00)$ & $10(50.00)$ & $6(30.00)$ \\
\hline
\end{tabular}




\begin{tabular}{|c|c|c|c|c|c|c|}
\hline & Gender & Poor & Low & Partial & Good & High \\
\hline & No of drugs & Poor & Low & Partial & Good & High \\
\hline \multirow{3}{*}{ Ch diseases } & $1 \mathrm{drug}$ & $10(20.83)$ & $38(79.16)$ & $0(0.00)$ & $0(0.00)$ & $0(0.00)$ \\
\hline & $2-4$ & $8(12.50)$ & $20(31.25)$ & $10(15.62)$ & $26(40.62)$ & $0(0.00)$ \\
\hline & $>4$ & $0(0.00)$ & $0(0.00)$ & $3(37.5)$ & $3(37.5)$ & $2(25.00)$ \\
\hline \multirow{4}{*}{ Cancers } & 1 drug & $1(5.00)$ & $1(5.00)$ & $4(20.00)$ & $6(30.00)$ & $8(40.00)$ \\
\hline & $2-4$ & $2(1.96)$ & $6(3.92)$ & $18(17.64)$ & $40(39.21)$ & $36(35.29)$ \\
\hline & $>4$ & $2(7.14)$ & $2(7.14)$ & $6(2.14)$ & $10(35.71)$ & $8(28.57)$ \\
\hline & Frequency & Poor & Low & Partial & Good & High \\
\hline \multirow{3}{*}{ Ch diseases } & od & $10(19.23)$ & $32(61.53)$ & $5(9.61)$ & $5(9.61)$ & $0(0.00)$ \\
\hline & bd & $6(16.66)$ & $10(27.77)$ & $9(25.00)$ & $9(25.00)$ & $2(5.55)$ \\
\hline & Tid & $4(12.5)$ & $14(43.75)$ & $7(21.87)$ & $7(21.87)$ & $0(0.00)$ \\
\hline \multirow{3}{*}{ Cancers } & od & $1(2.00)$ & $1(2.00)$ & $10(20.00)$ & $24(48.00)$ & $14(28.00)$ \\
\hline & bd & $3(5.76)$ & $3(5.76)$ & $10(19.23)$ & $16(30.76)$ & $20(38.46)$ \\
\hline & tid & $3(6.25)$ & $3(6.25)$ & $8(16.66)$ & $16(20.83)$ & $18(37.50)$ \\
\hline
\end{tabular}

In cancer disease group, $3.65 \%(\mathrm{n}=3)$ illiterate patients had poor, $8.53 \%(\mathrm{n}=7)$ low, 20.73\% $(\mathrm{n}=17)$ partial, 42.68\% ( $n=35)$ good, and $24.39 \%(n=20)$ high adherence. Among literate patients, $1.47 \%(\mathrm{n}=1)$ had poor, $4.41 \%$ $(n=3)$ low, $14.70 \%(n=10)$ partial and $32.35 \%(n=22)$ good and $47.05 \%(\mathrm{n}=32)$ high adherence. In chronic disease group, $27.77 \%(\mathrm{n}=25)$ patients from rural areas had poor, 56.66\% $(\mathrm{n}=51)$ low, 6.66\% $(\mathrm{n}=6)$ partial and 8.88\% $(n=8)$ good adherence. 33.33\% $(n=10)$ from urban areas had partial, $60.00 \%(\mathrm{n}=18)$ had good and $6.77 \%$ $(n=2)$ had high adherence. In cancer group, $2.00 \%(n=2)$ patients from rural areas had poor, $10.00 \%(\mathrm{n}=10)$ low, $8.00 \%(\mathrm{n}=8)$ partial, $50.00 \%(\mathrm{n}=50)$ good and $30.00 \%$ $(\mathrm{n}=30)$ high adherence. Among patients from urban areas $16.00 \%(\mathrm{n}=8)$ had partial, $40.00 \%(\mathrm{n}=20)$ good and $44.00 \%(n=22)$ high adherence. In chronic disease group, patients with treatment duration of less than one year, $25.00 \% \quad(n=5)$ had poor, and $75.00 \% \quad(n=15)$ low adherence. In subgroup 1-4 years treatment duration, 26.66\% ( $\mathrm{n}=16)$ had poor, 66.66\% $(\mathrm{n}=40)$ low, 3.33\% $(\mathrm{n}=2)$ partial and $3.33 \%(\mathrm{n}=2)$ good adherence. In subgroup of treatment duration of more than 4 years, $25.00 \%(\mathrm{n}=10)$ had partial, $70.00 \%(\mathrm{n}=28)$ good and $5.00 \%(n=2)$ high adherence. In cancer group, those with less than one year treatment, $3.12 \%(\mathrm{n}=1)$ had poor, $3.12 \%(\mathrm{n}=1)$ low, $12.50 \%(\mathrm{n}=4)$ partial, $50.00 \%(\mathrm{n}=16)$ good and $32.25 \%(\mathrm{n}=10)$ high adherence. In 1-4-year, treatment subgroup, $4.08 \%(n=4)$ had poor, $8.16 \%(n=8)$ had low, $10.20 \%(\mathrm{n}=10)$ partial, $40.81 \%(\mathrm{n}=40)$ good and $36.73 \%(\mathrm{n}=36)$ high adherence. In more than 4 years treatment sub-group, $20.00 \%(\mathrm{n}=4)$ had partial, $50.00 \%$ $(n=10)$ good, and $30.00 \%(n=6)$ high adherence. In chronic disease group, those taking one drug, 20.83\% $(n=10)$ had poor, and $79.16 \%(n=38)$ low adherence. Those taking 2-4 drugs, $12.50 \%(\mathrm{n}=8)$ had poor, $31.25 \%$ $(n=20)$ low, $15.62 \%(n=10)$ partial, and $40.62 \%(n=26)$ good adherence. In those patients taking more than 4 drugs, $37.5 \%(n=3)$ had partial, $37.5 \%(n=3)$ had good and $25.00 \%(\mathrm{n}=2)$ had high adherence. In cancer group, those taking one drug, $5.00 \%(\mathrm{n}=1)$ had poor, $5.00 \%$ $(\mathrm{n}=1)$ low, $20.00 \%(\mathrm{n}=4)$ partial, $30.00 \%(\mathrm{n}=6)$ good, and $40.00 \%(\mathrm{n}=8)$ high adherence. Those taking 2-4 drugs, $1.96 \%(n=2)$ had poor, 3.92\% $(n=6)$ low, $17.64 \%(n=18)$ partial, $39.21 \%(\mathrm{n}=40)$ good and $35.29 \%(\mathrm{n}=36)$ high adherence. Those taking more than 4 drugs, $7.14 \%(n=2)$ had poor, $7.14 \%(\mathrm{n}=2)$ low, $2.14 \%(\mathrm{n}=6)$ partial, $35.71 \%$ $(\mathrm{n}=10)$ good and $28.57 \%(\mathrm{n}=8)$ high adherence. In chronic disease group, those taking the drugs once a day, $19.23 \%$ $(n=10)$ had poor, 61.53\% (n=32) low, 9.61\% $(n=5)$ partial, and 9.61\% ( $\mathrm{n}=5)$ good adherence. Those taking twice a day $16.66 \%(\mathrm{n}=6)$ poor, $27.77 \%(\mathrm{n}=10)$ low, $25.00 \%(\mathrm{n}=9)$ partial, $25.00 \%(\mathrm{n}=9)$ good, and $5.55 \%$ $(\mathrm{n}=2)$ high adherence. Those taking the drugs thrice a day or more, $12.5 \%(\mathrm{n}=4)$ had poor, $43.75 \%(\mathrm{n}=14)$ low, $21.87 \%(\mathrm{n}=7)$ partial, and $21.87 \%(\mathrm{n}=7)$ good adherence. In cancer group, those taking the drugs once a day, $2.00 \%$ $(\mathrm{n}=1)$ had poor, $2.00 \%(\mathrm{n}=1)$ low, $20.00 \%(\mathrm{n}=10)$ partial, $48.00 \%(n=24)$ good and $28.00 \%(n=14)$ high adherence. Those taking the drugs twice a day, 5.76\% $(n=3)$ had poor, $5.76 \%(\mathrm{n}=3)$ low, $19.23 \%(\mathrm{n}=10)$ partial, $30.76 \%$ $(\mathrm{n}=16)$ good and $38.46 \%(\mathrm{n}=20)$ high adherence. Those taking the drugs thrice or more, $6.25 \%(\mathrm{n}=3)$ had poor, $6.25 \%(\mathrm{n}=3)$ low, 16.66\% $(\mathrm{n}=8)$ partial, $20.83 \%(\mathrm{n}=16)$ good and $37.50 \%(n=18)$ high adherence (Table 3$)$.

Table 4: Pearson correlations.

\begin{tabular}{|llll|}
\hline Variable pair & $\begin{array}{l}\text { Pearson } \\
(\mathbf{r})\end{array}$ & $\begin{array}{l}\text { Interpre } \\
\text { tation }\end{array}$ & $\begin{array}{l}\text { Signifi } \\
\text { cance }\end{array}$ \\
\hline $\begin{array}{l}\text { Gender and } \\
\text { adherence }\end{array}$ & 0.391 & Moderate & 0.000 \\
\hline Age \& adherence & 0.627 & Strong & 0.000 \\
\hline $\begin{array}{l}\text { Literacy and } \\
\text { adherence }\end{array}$ & 0.939 & Very strong & 0.000 \\
\hline $\begin{array}{l}\text { Location and } \\
\text { adherence }\end{array}$ & 0.351 & Moderate & 0.000 \\
\hline $\begin{array}{l}\text { Duration of } \\
\text { treatment and } \\
\text { adherence }\end{array}$ & 0.095 & Negligible & 0.247 \\
\hline $\begin{array}{l}\text { No. of drugs and } \\
\text { adherence }\end{array}$ & 0.088 & Negligible & 0.283 \\
\hline $\begin{array}{l}\text { Frequency of } \\
\text { administration } \\
\text { and adherence }\end{array}$ & 0.026 & Negligible & 0.752 \\
\hline
\end{tabular}


A moderate Pearson correlation was found between adherence and gender $(\mathrm{r}=0.391)$ and adherence and area of residence of the patient ( $\mathrm{r}=0.351)$. A strong correlation was found between adherence and age $(r=0.627)$. A very strong correlation was found between adherence and literacy $(\mathrm{r}=0.939)$. A negligible correlation was found between adherence and duration of therapy, number of drugs, and frequency of drug administration ( $\mathrm{r}=0.095$, $0.088,0.026$ ) (Table 4).

\section{DISCUSSION}

Patient adherence in chronic illnesses is low. In malignancies, although oral anticancer therapies offer many advantages, yet non-adherence may be a barrier to their effective use. ${ }^{10}$ In cancer patients despite higher motivation towards medication adherence, adherence ranges from $16 \%$ to $100 \% .^{11}$

In the present study we found that as compared to chronic diseases, patients suffering from cancers were much more adherent to medications. $74.66 \%$ of cancer patients had good or high adherence as compared to $21.66 \%$ patients suffering from chronic diseases.

We also found that females had slightly less medication adherence as compared to males. Bradi et al and Marie et al also found females less adherent. ${ }^{12,13}$

We found better medication adherence in young patients. But Alina et al found very young and very old less adherent. ${ }^{14}$ Katrine et al while reviewing age-related medication adherence found a statistically significant relationship between age and medication adherence: six studies demonstrated that increased age is correlated with higher medication adherence. ${ }^{15}$ We found literate people more adherent than illiterate. Sharon et al also found higher adherence in patients living in areas with higher education rates. ${ }^{16}$

We found poor adherence in patients from rural areas. The reason can be that the rural areas are medically underserved as compared to urban areas. The literacy rate is also lower in rural areas. Michelle YM et al also report similar findings in a study on medication adherence in rural low-income adults. ${ }^{17}$ Cody et al found that overall adherence rates between urban and rural adults were not significantly different. ${ }^{18} \mathrm{We}$ found that the prescription of a smaller number of drugs or lesser frequency of drug administration did not result in better adherence. Richard W Grant also found very high medication adherence rates in type 2 diabetes regardless of number of medicines prescribed. ${ }^{19}$

Though reducing the number of inappropriate medications is important, yet it should not be presumed that simply decreasing the number of medications will automatically improve adherence. ${ }^{20}$
The validity of our findings relies primarily on the accuracy of responses. The authors tried to minimize recall bias by using a well-structured pre-validated questionnaire. Another limitation of this study is the limited sample size.

The design of the study does not ensure that the study population is representative of all patients. We also did not analyze the data on the type of cancer, type of chronic illness, type of medication, and duration of illness. Therefore, the present study is a pilot study, illustrating only a portion of reality and providing a starting point for further, more extensive studies.

\section{CONCLUSION}

We found high medication adherence in cancer patients as compared to those having chronic ailments. Statistically, a significant correlation was found between gender, age, literacy, and area of residence of the participant patients. Male, literate, young patients, and those from urban areas had higher adherence as compared to opposite groups. But adherence was not complete in any subset of the study population. Adherence is a multifactorial phenomenon and there are numerous challenges in understanding the reasons for non-adherence. To derive the maximum benefit of prescribed therapy, various interventions to improve adherence have to be designed. Typically, adherence rates of $80 \%$ or more are needed for optimal therapeutic efficacy.

\section{ACKNOWLEDGEMENTS}

The authors would like to express their sincere thanks and gratitude to Dr. Atta Abbas Naqvi and Professor Mohamed Azmi Hassali, of Discipline of Social and Administrative Pharmacy, School of Pharmaceutical Sciences, Universiti Sans Malaysia (USM), Penang, Malaysia, for allowing to use GMAS in this study. The authors also wish to thank the patients who participated in the study.

\section{Funding: No funding sources}

Conflict of interest: None declared

Ethical approval: The study was approved by the Institutional Ethics Committee

\section{REFERENCES}

1. E. Sabaté, Adherence to Long-Term Therapies: Evidence for Action, World Health Organization, Geneva, Switzerland, 2003. Available at: https://www.who.int/chp/knowledge/publications/adh erence_full_report.pdf?ua=1. Accessed on 28 June 2020 .

2. Jimmy B, Jose J. Patient medication adherence: measures in daily practice. Oman Med J. 2011;26(3):155.

3. Cutler RL, Fernandez-Llimos F, Frommer M, Benrimoj C, Garcia-Cardenas V. Economic impact of 
medication non-adherence by disease groups: a systematic review. Br Med J. 2018;8(1):e016982.

4. Partridge AH, Avorn J, Wang PS, Winer EP. Adherence to therapy with oral antineoplastic agents. J Nat Cancer Instit. 2002;94(9):652-61.

5. Foulon V, Schöffski P, Wolter P. Patient adherence to oral anticancer drugs: an emerging issue in modern oncology. Acta Clin Belg. 2011;66(2):85-96

6. Gruszczyńska M Sosnowska MB,Szemik S.Selected psychological aspects and medication adherence in oncological patients.Cancer Med. 2020;9(3):943-50.

7. Culig J, Leppée M. From Morisky to Hill-bone; selfreports scales for measuring adherence to medication. Collegium Antropologicum. 2014;38(1):55-62.

8. Osterberg L, Blaschke T. Adherence to medication, N Engl J Med. 2005;353:487-97.

9. Naqvi AA, Hassali MA, Rizvi M, Zehra A, Iffat W, Haseeb A, et al. Development and validation of a novel General Medication Adherence Scale (GMAS) for chronic illness patients in Pakistan. Frontier Pharmacol. 2018;9:1124.

10. Boyle D. Enhancing patient adherence to improve outcomes with oral chemotherapy proceedings from a symposium at the 22007 hematology/oncology pharmacy association annual conference. US Pharm 2007;32:1-8

11. Foulon V, Schöffski P, Wolter P. Patient adherence to oral anticancer drugs: an emerging issue in modern oncology. Acta Clin Belg. 2011;66(2):85-96.

12. Granger BB Ekman I Granger CB Ostergren J Olofsson B Michelson E et al. Adherence to medication according to sex and age in the CHARM programme Eur J Heart Fail. 2009; 11:1092-8.

13. Manteuffel M, Williams S, Chen W, Verbrugge RR, Pittman DG, Steinkellner A. Influence of patient sex and gender on medication use, adherence, and prescribing alignment with guidelines. J Wom Heal. 2014;23(2):112-9.

14. Gast A, Mathes T. Medication adherence influencing factors - an (updated) overview of systematic Reviews. Syst Rev. 2019;8:112.

15. Krueger K, Botermann L, Schorr SG, GrieseMammen N, Laufs U, Schulz M. Age-related medication adherence in patients with chronic heart failure: a systematic literature review. Int J Cardiol. 2015;184:728-35.

16. Rolnick SJ, Pawloski PA, Hedblom BD, Asche SE, Bruzek RJ . Patient characteristics associated with medication adherence. Clin Med Res 2013;11:54-65.

17. Martin MY, Kim YI, Kratt P, Litaker MS, Kohler CL, Schoenberger YM, et al. Medication adherence among rural, low-income hypertensive adults: a randomized trial of a multimedia community-based intervention. Am J Health Promot. 2011;25:372-8.

18. Arbuckle C, Tomaszewski D, Aronson BD, Brown L, Schommer J, Morisky D, et al. Evaluating factors impacting medication adherence among rural, urban, and suburban populations. J Rural Health. 2018

19. Grant RW, Devita NG, Singer DE. Polypharmacy and medication adherence in patients with type 2 diabetes. Diabet Car. 2003;26:1408-12

20. Murray MD, Kroenke K. Polypharmacy and medication adherence: small steps on a long road. J Gen Intern Med. 2001;16:137-9.

Cite this article as: Mir SA, Shakeel D. Prevalence and predictors of medication non-adherence in some common non-communicable chronic diseases and cancers: a comparative study. Int J Basic Clin Pharmacol 2020;9:1681-7. 